\title{
Synergistic antitumor effects of the combined treatment with an HDAC6 inhibitor and a COX-2 inhibitor through activation of PTEN
}

\author{
GUANHUA ZHANG $^{1,2}$ and YE-HUA GAN ${ }^{1,2}$ \\ ${ }^{1}$ Central Laboratory and ${ }^{2}$ Department of Oral and Maxillofacial Surgery, \\ Peking University School and Hospital of Stomatology, Haidian, Beijing 100081, P.R. China
}

Received March 15, 2017; Accepted August 11, 2017

DOI: $10.3892 /$ or.2017.5981

\begin{abstract}
Chemotherapy is one of the most effective nonsurgical treatments for various types of tumor. Identifying different combinations of antitumor agents that can produce synergistic antitumor effects remains an important clinical strategy. In the present study, we showed that the combination of histone deacetylase 6 (HDAC6) inhibitor tubastatin A together with cyclooxygenase-2 (COX-2) inhibitor celecoxib resulted in synergistic antitumor effects in CAL 27 and SACC-83 cells. Treatment with celecoxib alone promoted the membrane translocation of phosphatase and tensin homolog (PTEN), indicating PTEN activation, and consequently led to protein kinase $\mathrm{B}$ (AKT) dephosphorylation (inactivation). Similarly, treatment with an HDAC6 inhibitor alone promoted PTEN membrane translocation and correspondingly dephosphorylated AKT. The combination of celecoxib and an HDAC6 inhibitor synergistically increased PTEN membrane translocation and inactivated AKT. Moreover, celecoxib enhanced the HDAC6 inhibitor-induced antitumor effects in PTEN-deficient U-87 MG cells that had been stably transfected with wild-type PTEN, but not in the same cell line stably transfected with mutant PTEN-K163R, which cannot be activated by HDAC6 inhibitors. In summary, the results indicated that the COX-2 inhibitor celecoxib enhanced the HDAC6 inhibitor-induced antitumor effects by activating the PTEN/AKT signaling pathway.
\end{abstract}

Correspondence to: Dr Ye-Hua Gan, Central Laboratory, Peking University School of Stomatology, 22 Zhongguancun Avenue South, Haidian, Beijing 100081, P.R. China

E-mail: kqyehuagan@bjmu.edu.cn

Key words: PTEN, COX-2, AKT, PGE2, HDAC, HDAC6 inhibitor, celecoxib

\section{Introduction}

Chemotherapy is one of the major strategies for cancer treatment, and functions by targeting the physiological characteristics of cancer cells, including proliferation, angiogenesis, apoptosis, invasion and migration (1). However, drug resistance (2) and severe side-effects (3) still hinder the effects of chemotherapy. The use of drug combinations has numerous benefits for cancer therapy. It enhances the therapeutic effects or decreases the required dosages of each drug thereby reducing the severity of adverse effects (4). Thus, the search for combinations of agents that can achieve synergistic antitumor effects remains an important strategy with which to improve the effects of chemotherapy.

Acetylation is a major form of protein post-translational modification and is responsible for regulating various cellular processes, including cell proliferation and cell survival (5). Acetylation is catalyzed by histone acetyltransferases (HATs) and histone deacetylases (HDACs). HATs transfer acetyl groups to lysine residues, whereas HDACs remove acetyl groups from lysine residues (6). In total, 18 human HDACs have been identified, which are categorized into four classes: Class I (HDAC1, HDAC2, HDAC3 and HDAC8), IIa (HDAC4, HDAC5, HDAC7 and HDAC9), IIb (HDAC6 and 10) and IV (HDAC11) are classical HDACs, whose activities are inhibited by pan-HDAC inhibitors, such as trichostatin A; whereas class III HDACs, also known as sirtuins, are not affected by trichostatin A (7).

HDACs are overexpressed in many types of tumors, and inhibition of HDACs can result in the inhibition of cell proliferation and induction of apoptosis (8). HDAC inhibitors can achieve antitumor effects through the phosphatase and tensin homolog (PTEN)/protein kinase B (AKT) signaling pathway $(9,10)$. To date, three pan-HDAC inhibitors, namely vorinostat (SAHA), belinostat (PXD-101) and panobinostat (LBH-589), have been approved by the US Food and Drug Administration (FDA) for the treatment of cancer (11). Clinical application of pan-HDAC inhibitors can result in several adverse side-effects, such as fatigue, diarrhea, bone marrow toxicity and thrombocytopenia (12). This is likely due to the fact that most HDACs are necessary for important biological processes; knockout of HDAC1, or most other 
HDACs (except HDAC6), leads to death or severe defects during embryonic development or shortly after birth (13). However, HDAC6-deficient mice typically survive (14), and the inhibition of HDAC6 has antitumor effects (15). Aberrant expression of HDAC6 is an independent prognostic indicator in human breast cancer (16). Moreover, our previous study demonstrated that the activation of PTEN through K163 acetylation, in response to the inhibition of HDAC6, is an important mechanism underlying the antitumor effects of pan-HDAC inhibitors (17). Therefore, HDAC6-specific inhibitors may have potential clinical advantages as antitumor agents as they do not affect other critical HDACs.

PTEN is a tumor-suppressor gene that plays a crucial role in cell growth, development and apoptosis (18). PTEN function is commonly lost by deletion or mutation in many types of human cancer, including melanoma, pancreatic, colorectal and lung cancer (19). PTEN inhibits cell growth and proliferation by removing a phosphate group from PIP3, thus preventing the activation of AKT (20). Membrane-bound PTEN is the activated form of the protein $(21,22)$.

Cyclooxygenase-2 (COX-2) is an important rate-limiting enzyme in the process of prostaglandin E2 (PGE2) synthesis. PGE2 induces proliferation, invasion and migration of cancer cells via several signaling pathways, including the $\beta$-catenin and AKT pathways $(23,24)$. COX-2 is overexpressed in many different types of cancer (25). Inhibition of COX-2 induces the inhibition of proliferation and apoptosis in several cancer cell lines, including colorectal, prostate and breast cancer cell lines (26). These studies suggest that COX-2 may be a potential novel therapeutic target for chemotherapy. The COX-2 selective inhibitor celecoxib, alone or in combination with other agents, is currently being clinically tested in lung cancer patients (27). Although COX-2 inhibitors enhance the anti-angiogenic effects of pan-HDAC inhibitors (28), it is not known which HDAC is involved in the crosstalk with the COX-2 signaling pathway. We previously demonstrated that COX-2 inhibitors upregulate PTEN (22), and HDAC6 inhibitors can also activate PTEN (17). This prompted the question of whether COX-2 inhibitors can enhance the antitumor effects of HDAC6 inhibitors through the synergistic activation of PTEN.

In the present study, celecoxib, a COX-2 inhibitor, was shown to enhance the antitumor effects of an HDAC6 inhibitor through synergistic activation of PTEN and inactivation of AKT in CAL 27 and SACC-83 cells.

\section{Materials and methods}

Cell culture and treatments. CAL 27 cells derived from human tongue squamous cell carcinoma (29) were cultured in Dulbecco's modified Eagle's medium (DMEM; Gibco, Grand Island, NY, USA) with 10\% fetal bovine serum (FBS) at $37^{\circ} \mathrm{C}$ in an atmosphere with $5 \% \mathrm{CO}_{2}$. SACC- 83 cells derived from human salivary adenoid cystic cancer (30), were cultured in RPMI-1640 medium (Gibco) with $10 \% \mathrm{FBS}$ at $37^{\circ} \mathrm{C}$ with $5 \% \mathrm{CO}_{2}$. U-87 MG cells that had been stably transfected with wild-type PTEN and PTEN-K163R were incubated in DMEM with $10 \% \mathrm{FBS}$ at $37^{\circ} \mathrm{C}$ with $5 \% \mathrm{CO}_{2}$. Cells were treated with various doses of tubastatin A or celecoxib alone for $24 \mathrm{~h}$, or with a combination of an HDAC6 inhibitor (tubastatin A or tubacin) and celecoxib for $24 \mathrm{~h}$.
Reagents and antibodies. Tubastatin A, tubacin and celecoxib were purchased from Selleck Chemicals (Houston, TX, USA). The anti-PTEN (\#9552) and anti-phospho-AKT (S473; \#4058) antibodies were purchased from the Cell Signaling Technology, Inc. (Danvers, MA, USA), and the anti- $\beta$-actin (I-19) antibody (TA-09) was purchased from ZSGB-BIO Co. (Beijing, China).

Protein extraction and western blotting. Whole cell lysates were extracted with RIPA lysis buffer (Applygen Technologies Inc., Beijing, China). Membrane proteins were extracted using a Nuclear-Cytosol Extraction kit (Applygen Technologies Inc.). Protein concentrations were determined via BCA protein assay (Thermo Fisher Scientific, Inc., Waltham, MA USA). Equal amounts of samples were subjected to $10 \%$ sodium dodecyl sulfate-polyacrylamide gel electrophoresis, and then transferred to a polyvinylidene fluoride membrane (EMD Millipore, Billerica, MA, USA). The membrane was blocked with fat-free milk (5\%) in TBS-T $(50 \mathrm{mmol} / 1 \mathrm{Tris}$, $\mathrm{pH} 7.5 ; 150 \mathrm{mmol} / 1 \mathrm{NaCl} ; 0.05 \%$ Tween-20) for $1 \mathrm{~h}$. Following incubation with the primary antibodies (diluted 1:1,000 in TBS-T) overnight at $4^{\circ} \mathrm{C}$, the membrane was washed extensively with TBS-T three times ( $5 \mathrm{~min} /$ wash) at room temperature and then incubated with a secondary antibody conjugated with a fluorophore for $1 \mathrm{~h}$ at room temperature. After extensive washing with TBS-T, the protein bands on the membrane were visualized with an Odyssey infrared imaging system (Odyssey; LI-COR, Lincoln, NE, USA).

Cell proliferation assay. The cell proliferation assay was performed using Cell Counting Kit-8 (CCK-8; Dojindo, Kumamoto, Japan) according to the manufacturer's instructions. In brief, the cells were seeded into 96 -well plates $\left(1.5 \times 10^{3}\right.$ cells/well $)$ and treated with $20 \mu \mathrm{M}$ celecoxib or $20 \mu \mathrm{M}$ HDAC6 inhibitor (either tubastatin A or tubacin), or a combination of $20 \mu \mathrm{M}$ COX-2 inhibitor and $20 \mu \mathrm{M}$ HDAC6 inhibitor for $24 \mathrm{~h}$. Subsequently, $10 \mu \mathrm{l}$ CCK- 8 reagent was added to each well. Cells were further incubated at $37^{\circ} \mathrm{C}$ for $3 \mathrm{~h}$, then the absorbance at $450 \mathrm{~nm}\left(\mathrm{OD}_{450}\right)$ of each well was measured. Data are presented as the mean \pm standard deviation (SD) of at least three independent experiments.

Analysis of drug interaction in vitro. The coefficient of drug interaction (CDI) was used to analyze the synergistic inhibitory effect of the drug combination. CDI was calculated as follows: $\mathrm{CDI}=\mathrm{AB} /(\mathrm{A} \times \mathrm{B}) . \mathrm{AB}$ is the $\mathrm{OD}_{450}$ ratio of the twodrug combination group to the control group; $\mathrm{A}$ and $\mathrm{B}$ are the $\mathrm{OD}_{450}$ ratios of each of the single-drug groups to the control group. $\mathrm{CDI}<1$ indicates a synergistic effect, $\mathrm{CDI}<0.7$ indicates a significant synergistic effect, $\mathrm{CDI}=1$ indicates additivity, and $\mathrm{CDI}>1$ indicates antagonism (31).

Assessment of cell apoptosis. Cells were washed in phosphate-buffered saline (PBS) three times, fixed with $10 \%$ formaldehyde for $5 \mathrm{~min}$, and then incubated with $5 \mathrm{mg} /$ $\mathrm{ml}$ 4,6-diamidino-2-phenylindole dihydrochloride (DAPI) in the dark for $5 \mathrm{~min}$ at room temperature. Following three washes in PBS, the cells were examined under a fluorescence microscope (Nikon Corporation, Tokyo, Japan). Apoptotic cells were considered to be those that presented features of nuclear condensation and fragmentation under fluorescence 
microscopy. Apoptotic cells were counted within five randomly selected fields, and the rate of apoptotic cells is presented as the mean \pm SD of at least three independent experiments.

Caspase-3/-7 activities were measuredusing Caspase-Glo ${ }^{\circledR} 3 / 7$ assay (Promega Corporation, Madison, WI, USA) according to the manufacturer's instructions. The cells were seeded into 96-well plates $\left(1.5 \times 10^{3}\right.$ cells/well $)$ and treated with $20 \mu \mathrm{M}$ celecoxib or $20 \mu \mathrm{M}$ HDAC6 inhibitor (either tubastatin A or tubacin), or the combination of COX-2 and HDAC6 inhibitors for $24 \mathrm{~h}$. Subsequently, the cells were separately transferred into a white-walled 96-well plate (100 $\mu \mathrm{l} /$ well). Caspase-Glo ${ }^{\circledR}$ 3/7 reagent (100 $\mu \mathrm{l}$; prepared prior to the start of the assay) was added to each well, which contained $100 \mu \mathrm{l}$ blank (culture medium without cells) or treated cells in culture medium. The plate was gently shaken using a plate shaker at $300 \mathrm{rpm}$ for $30 \mathrm{sec}$, incubated at room temperature for up to $3 \mathrm{~h}$, and then measured for the luminescence of each well on an EnSpire Multimode Plate Reader with Epic ${ }^{\circledR}$ Label-Free technology (PerkinElmer, Bridgeville, PA, USA).

Transwell migration and invasion assays. Cell migration and invasion assays were performed in Transwell chambers (Corning Costar, Corning, NY, USA) with polycarbonate membranes. For the migration assay, cells were seeded into the upper chambers of each well $\left(1 \times 10^{5}\right.$ cells/well $)$ in serum-free culture medium, while culture medium containing $10 \%$ FBS was added to the lower chambers. The cells were incubated for $12 \mathrm{~h}$, after which the cells on the top surface of the membrane were wiped off, and the cells on the bottom surface were fixed with $4 \%$ paraformaldehyde and stained with $0.01 \%$ crystal violet. Cells on the bottom surface of the membrane were examined under a light microscope, counted and averaged from six randomly selected fields.

The Transwell invasion assay was performed in the same manner as the migration assay, except that the upper chambers were coated with $20 \mu \mathrm{g}$ extracellular matrix gel (Sigma-Aldrich, St. Louis, MO, USA) prior to seeding the cells.

Xenograft tumor inoculation. BALB/c nude mice (6 weeks of age) were purchased from the Beijing Vital River Laboratory Animal Technology Co. Ltd. (Beijing, China). The care and treatment of experimental animals followed institutional guidelines. Mice were randomly allocated to four groups ( $n=4 /$ group). CAL 27 cells were subcutaneously inoculated $\left(5 \times 10^{6}\right.$ cells/mouse) into the left axilla of each mouse. After 10 days, each group received daily celecoxib $(40 \mathrm{mg} / \mathrm{kg}$ dissolved in methylcellulose) alone via gavage, or tubastatin A $(0.5 \mathrm{mg} / \mathrm{kg}$ dissolved in dimethyl sulfoxide) alone via intraperitoneal injection, or a combination of celecoxib $(40 \mathrm{mg} / \mathrm{kg}$ ) and tubastatin A $(0.5 \mathrm{mg} / \mathrm{kg})$, or their dissolvents, via gavage or intraperitoneal injection, for three weeks. The mice were then euthanized, and the weights of the xenograft tumors were measured.

Statistical analysis. All statistical examinations were performed using SPSS 21 for Windows (SPSS, Inc., Chicago, IL, USA). All data are presented as the mean \pm SD. Differences between multiple groups were analyzed by one-way analysis of variance. $\mathrm{P}<0.05$ was considered to indicate a statistically significant difference.

\section{Results}

Combined treatment with an HDAC6 inhibitor and a COX-2 inhibitor exerts synergistic effects on the proliferation, migration and invasion of CAL 27 cells. To examine whether the combination of HDAC6 and COX-2 inhibitors achieves synergistic effects on cell proliferation, migration and invasion, CAL 27 cells were treated with $20 \mu \mathrm{M}$ celecoxib alone, $20 \mu \mathrm{M}$ tubastatin $\mathrm{A}$ alone, or a combination of celecoxib and tubastatin A for $24 \mathrm{~h}$. As shown in Fig. 1A-C, following treatment of the cells with celecoxib or tubastatin A alone, cell proliferation, migration and invasion were slightly inhibited. The combined-treatment group showed synergistic inhibition of proliferation, and inhibition of cell migration and invasion. The CDI of the HDAC6 inhibitor tubastatin A combined with the COX-2 inhibitor celecoxib was calculated as 0.32 in CAL 27 cells, which indicated a significant synergistic effect.

Combined treatment with HDAC6 and COX-2 inhibitors exerts synergistic effects on the proliferation, migration and invasion of SACC-83 cells. To examine whether the synergistic effects on cell proliferation, migration and invasion are achieved by combining an HDAC6 inhibitor with a COX-2 inhibitor in SACC-83 cells, the cells were treated with $20 \mu \mathrm{M}$ celecoxib alone, $20 \mu \mathrm{M}$ tubastatin $\mathrm{A}$ alone, or a combination of celecoxib and tubastatin A for $24 \mathrm{~h}$. As shown in Fig. 2A-C, proliferation, cell migration and invasion were slightly inhibited in the cells treated with celecoxib or tubastatin A alone whereas the combined-treatment group showed synergistic inhibition of proliferation, and inhibition of cell migration and invasion. The CDI of the combined treatment was calculated as 0.51 in SACC-83 cells, which indicated a significant synergistic effect.

Apoptosis is induced following treatment with tubastatin A and/or celecoxib. DAPI staining and caspase-3/-7 activity were measured to detect the induction of apoptosis by tubastatin A alone, celecoxib alone and the combination of tubastatin A and celecoxib in CAL 27 and SACC-83 cells. The cells were treated with $20 \mu \mathrm{M}$ celecoxib alone, $20 \mu \mathrm{M}$ tubastatin A alone, or a combination of celecoxib and tubastatin A for $24 \mathrm{~h}$. As shown in Fig. 3A-D, treatment with celecoxib or tubastatin A alone led to a slight induction of cell apoptosis and marginal upregulation of caspase-3/-7 activity, whereas the combinedtreatment group showed significant induction of cell apoptosis and significant upregulation of caspase-3/-7 activity.

Treatment with celecoxib or tubastatin A activates PTEN and inactivates $A K T$. To understand the mechanism underlying the synergistic antitumor effects of the combination of celecoxib and tubastatin A, we evaluated the levels of membrane-bound PTEN and phospho-AKT in the cells after treatment with celecoxib for $24 \mathrm{~h}$. As shown in Fig. 4A, membrane-bound PTEN and total PTEN were dose-dependently upregulated, whereas phosphorylation of AKT was dose-dependently downregulated in the CAL 27 and SACC-83 cells. Subsequently, the levels of membrane-bound PTEN and phospho-AKT following treatment with tubastatin A for $24 \mathrm{~h}$ were evaluated. As shown in Fig. 4B, tubastatin A induced the dose-dependent upregulation of membrane-bound PTEN, but did not affect 
A

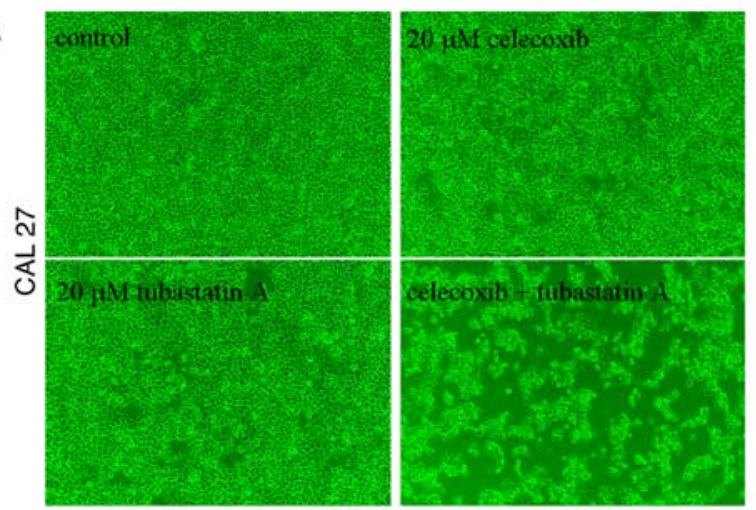

B

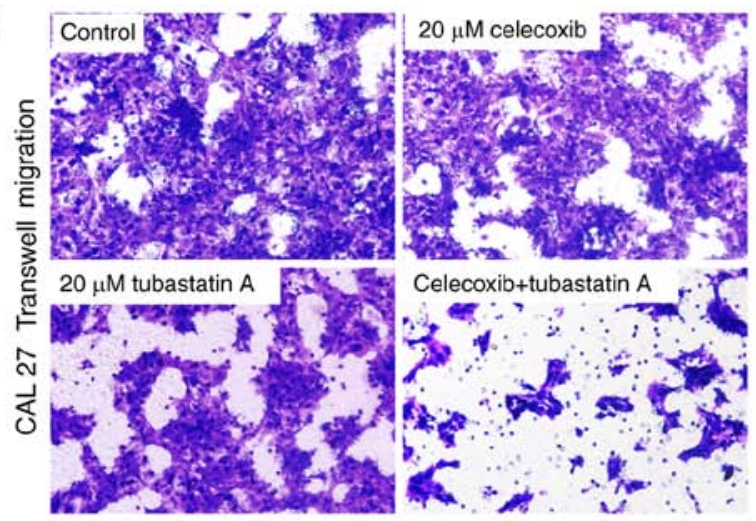

C

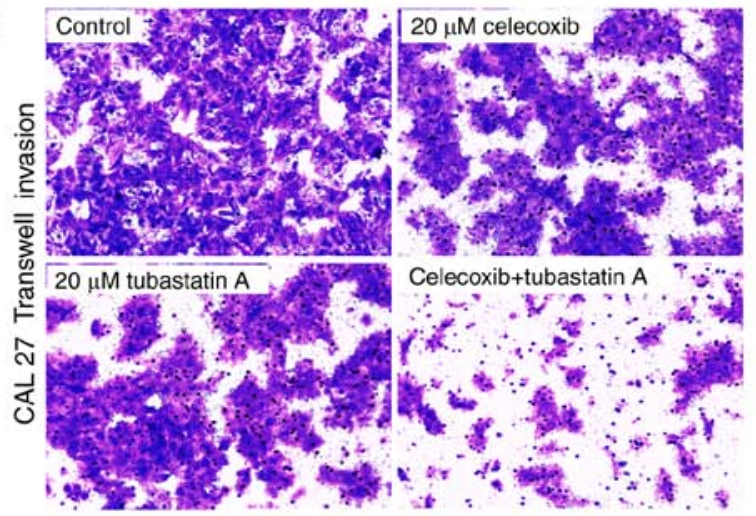

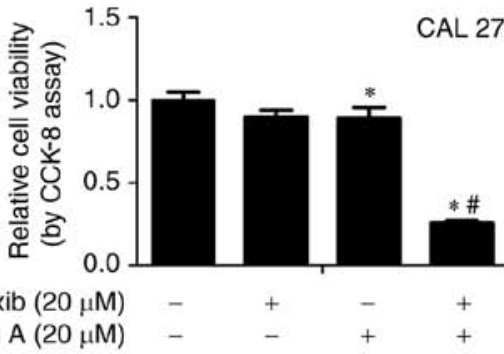

$\begin{array}{rlllll}\text { Celecoxib }(20 \mu \mathrm{M}) & - & & + & - & + \\ \text { Tubastatin A }(20 \mu \mathrm{M}) & - & & - & + & +\end{array}$

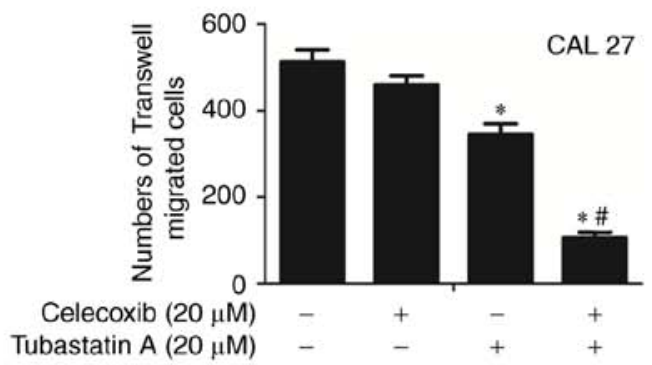

Tubastatin A $(20 \mu \mathrm{M})$

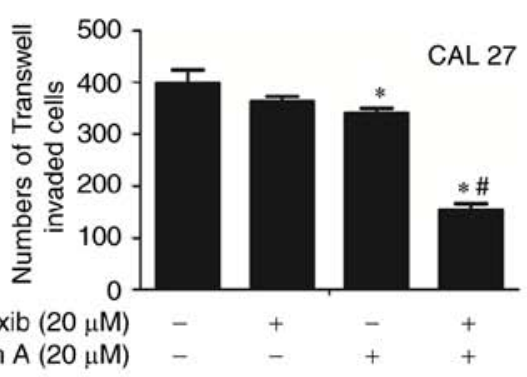

Figure 1. Synergistic effects of the combined treatment with an HDAC6 inhibitor and a COX-2 inhibitor on CAL 27 cell proliferation, migration and invasion. CAL 27 cells were treated with an HDAC6 inhibitor and/or a COX-2 inhibitor. (A) Phase-contrast photomicrographs of CAL 27 cells. Cell proliferation was evaluated by CCK-8 assay after the different treatments. (B) Transwell migration assay of CAL 27 cells after the different treatments. (C) Transwell invasion assay of CAL 27 cells after the different treatments. All data are presented as the mean \pm SD of at least three independent experiments; ${ }^{*}<0.05$ vs. control group; ${ }^{\text {"}} \mathrm{P}<0.05$ vs. celecoxib or tubastatin A groups $(n=3)$.

total PTEN expression in the CAL 27 and SACC-83 cells. Correspondingly, phospho-AKT was dose-dependently downregulated by tubastatin $\mathrm{A}$.

Combined treatment with an HDAC6 inhibitor and COX-2 inhibitor exerts synergistic effects on PTEN and AKT. Finally, we examined whether the combination of celecoxib and the HDAC6 inhibitor tubastatin A affects the activation of PTEN and inactivation of AKT in the CAL 27 and SACC-83 cells. As shown in Fig. 5A and B, celecoxib or tubastatin A treatments alone slightly upregulated membrane-bound PTEN and downregulated phospho-AKT, whereas the combination of celecoxib and tubastatin A synergistically upregulated membrane-bound PTEN and correspondingly downregulated phospho-AKT. Similar results were observed when the cells were treated with combined celecoxib and tubacin (another HDAC6 inhibitor). These results suggest that the synergistic antitumor effects achieved by the combination of an HDAC6 inhibitor and a COX-2 inhibitor may be due, at least partially, to the activation of PTEN and inactivation AKT.

Combined treatment with celecoxib and tubastatin A does not induce synergistic inhibition of proliferation in PTEN-deficient U-87 MG cells. To further examine whether 
A
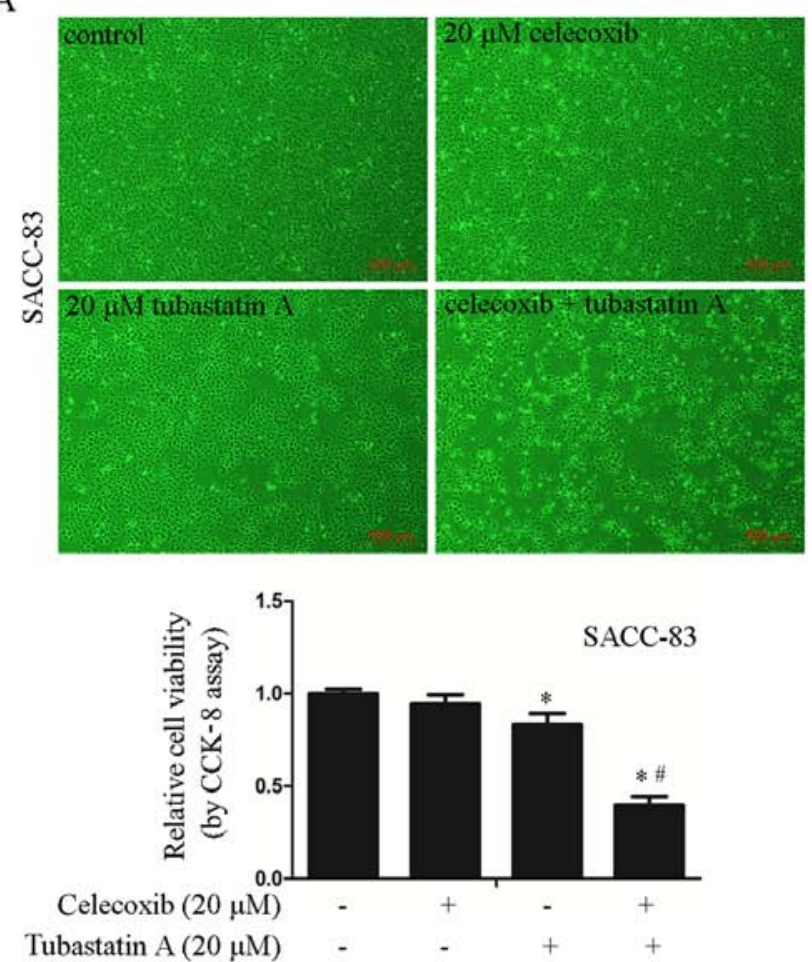

Tubastatin $\mathrm{A}(20 \mu \mathrm{M})$

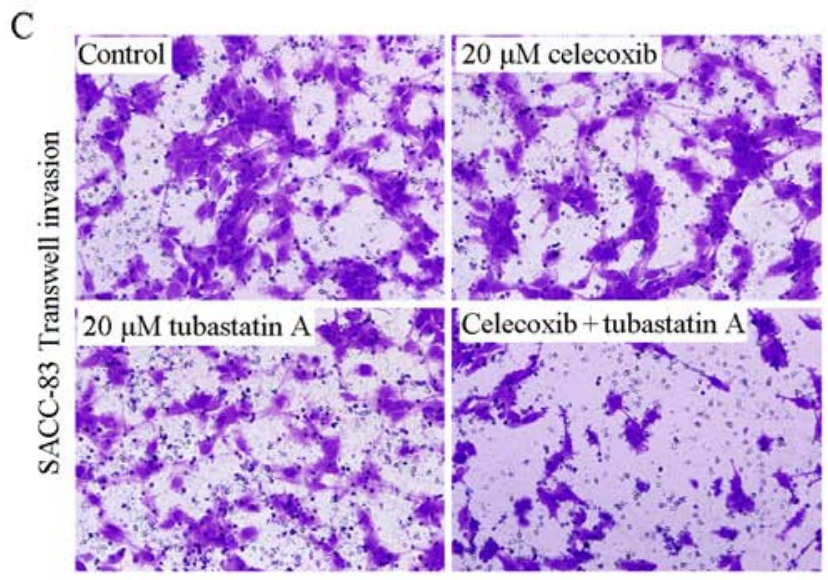

B
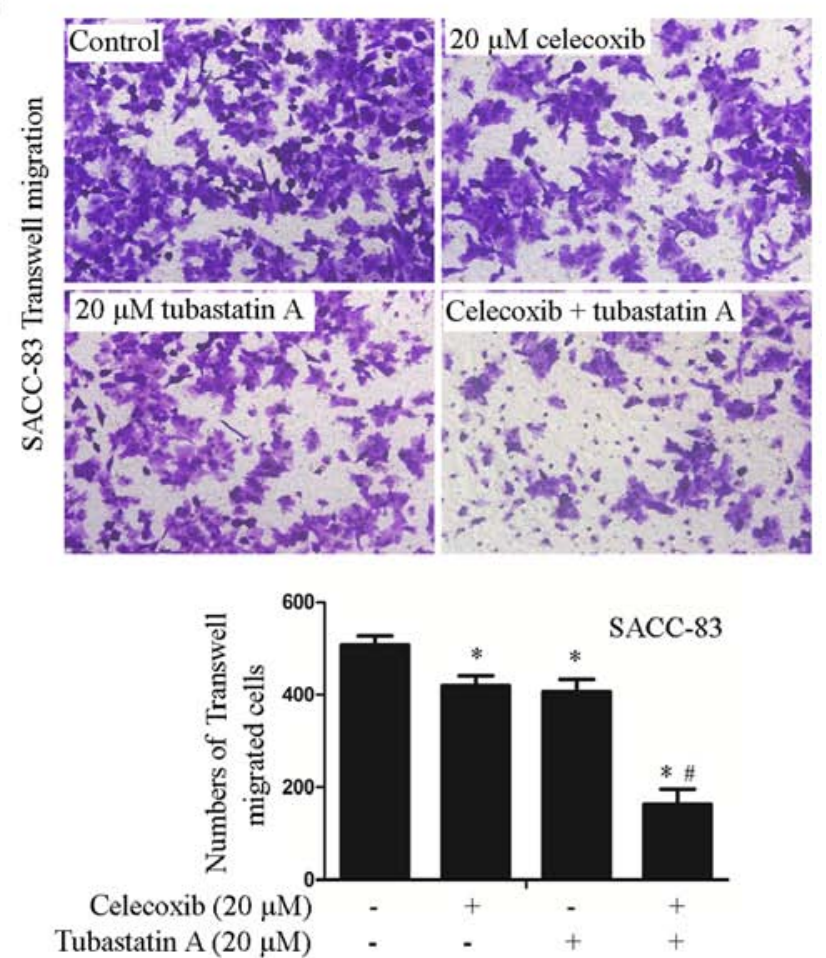

Tubastatin $\mathrm{A}(20 \mu \mathrm{M})$

Figure 2. Synergistic effects of the combined treatment with an HDAC6 inhibitor and a COX-2 inhibitor on SACC-83 cell proliferation, migration and invasion. SACC-83 cells were treated with an HDAC6 inhibitor and/or a COX-2 inhibitor. (A) Phase-contrast photomicrographs of SACC-83 cells after the different treatments. Cell proliferation was evaluated by CCK-8 assay after the different treatments. (B) Transwell migration assay of SACC-83 cells after the different treatments. (C) Transwell invasion assay of SACC-83 cells after the different treatments. All data are presented as the mean \pm SD of at least three independent experiments; ${ }^{\mathrm{P}}<0.05$ vs. the control group; ${ }^{\mathrm{P}}<0.05$ vs. the celecoxib or tubastatin A groups $(\mathrm{n}=3)$.

the PTEN/AKT signaling pathway is required for the synergistic antitumor effects of celecoxib and tubastatin A, PTEN-deficient U-87 MG cells were treated with $20 \mu \mathrm{M}$ celecoxib alone, or $20 \mu \mathrm{M}$ tubastatin $\mathrm{A}$ alone or the two combined. As shown in Fig. 6A and B, the combined treatment did not significantly affect proliferation $(\mathrm{CDI}=1.0)$. Similarly, the level of phospho-AKT in the combined-treatment group was not different from the level noted in the celecoxib alone, tubastatin A alone, or control groups (Fig. 6C).

Combined treatment with celecoxib and tubastatin A synergistically inhibits the proliferation of $U-87 M G$ cells stably transfected with wild-type PTEN, but not with mutant PTEN-K163R. In our previous study, inhibition of HDAC6 was shown to activate PTEN via PTEN acetylation at K163 (17). To examine whether the synergistic antitumor effects of the combined treatment with tubastatin A and celecoxib were dependent on PTEN acetylation at K163, U-87 MG cells were stably transfected with wild-type PTEN and mutant PTEN-K163R (with lysine replaced by arginine), and subsequently treated for $24 \mathrm{~h}$ with celecoxib alone, tubastatin A alone, or the two agents combined. As shown in Fig. 7A, synergistic inhibition of proliferation was achieved by the combination of tubastatin A and celecoxib in the U-87 MG cells stably transfected with wild-type PTEN $(\mathrm{CDI}=0.6)$, but not in the cells stably transfected with the 

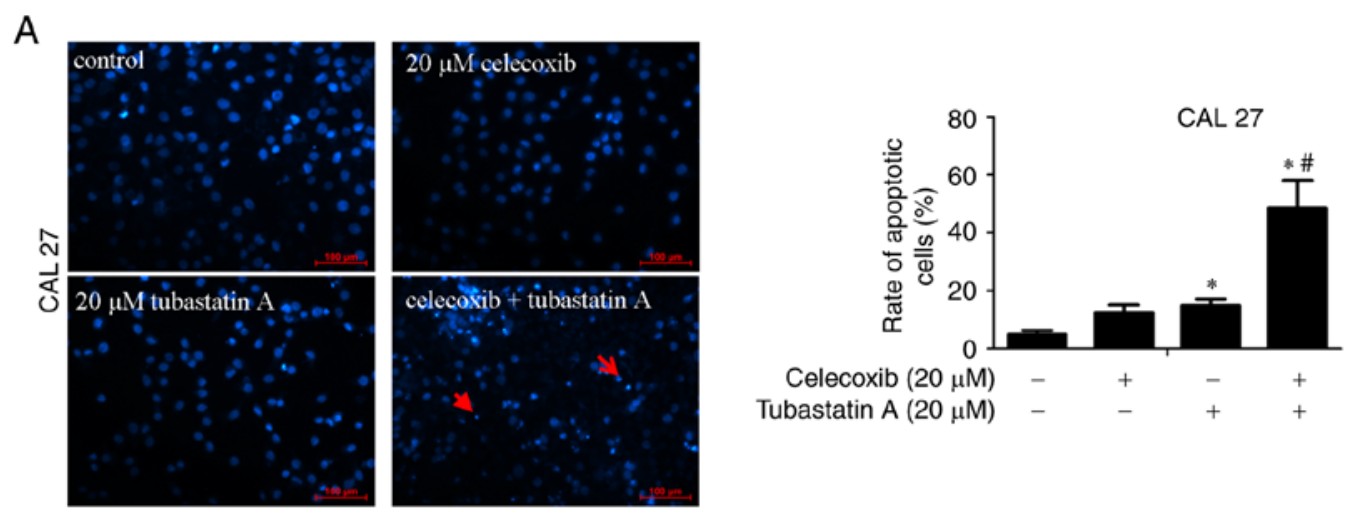

B
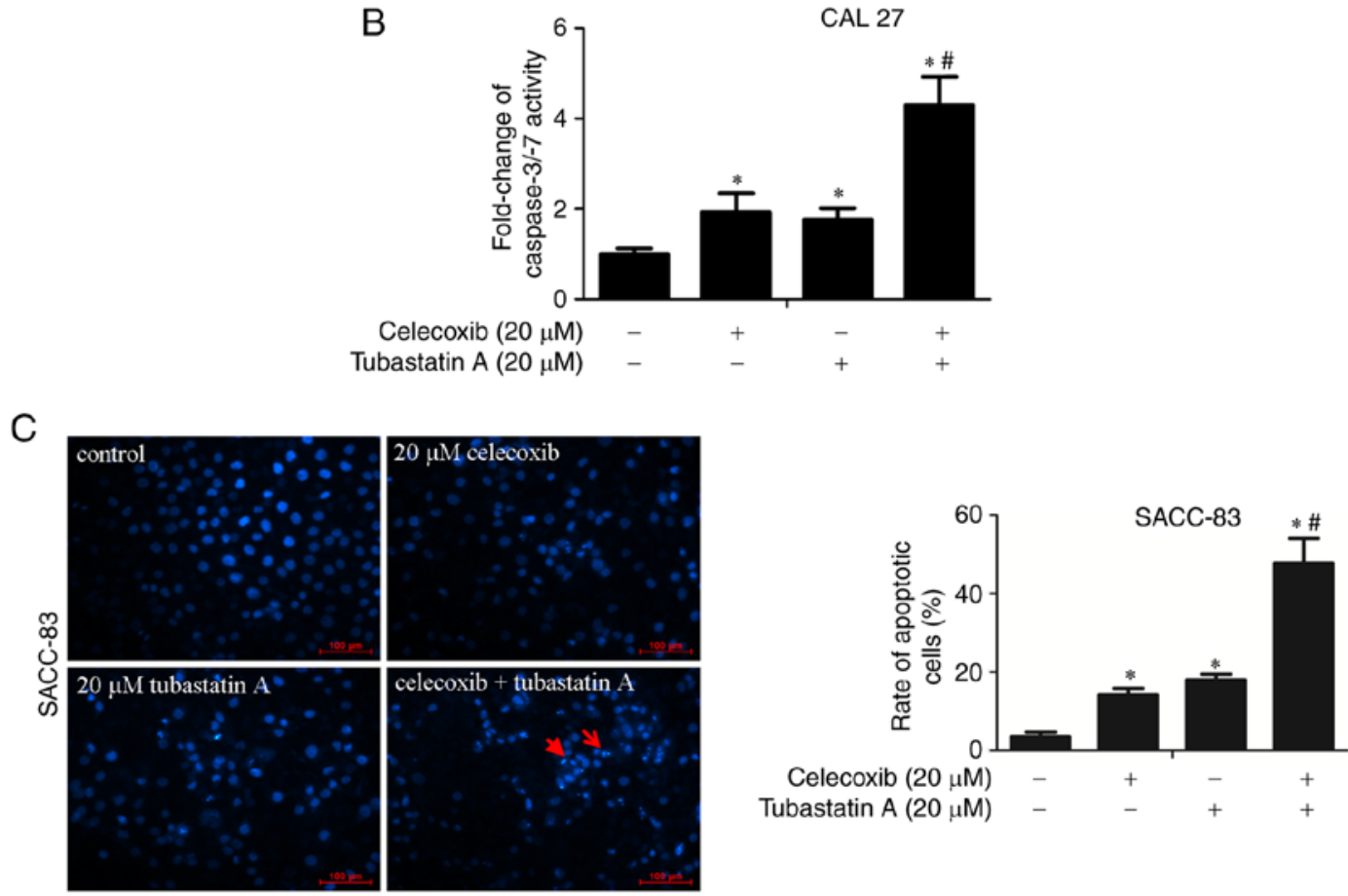

Tubastatin $\mathrm{A}(20 \mu \mathrm{M})$

SACC-83

$\mathrm{D}$

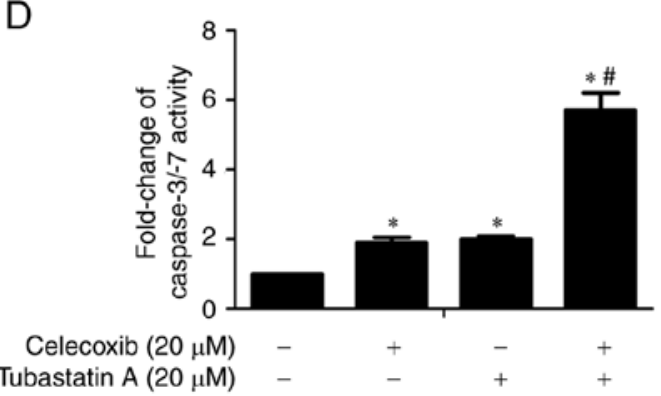

Figure 3. Synergistic effects of the combined treatment with an HDAC6 inhibitor and a COX-2 inhibitor on apoptosis. (A) Fluorescent photomicrographs of DAPI-stained CAL 27 cells after the different treatments. Apoptotic cells, exhibiting nuclear condensation (arrows with triangle) or fragmentation (arrows), were observed. (B) Caspase-3/-7 activity of CAL 27 cells after the different treatments. (C) Fluorescent photomicrographs of DAPI-stained SACC-83 cells. Apoptotic cells, exhibiting nuclear condensation (arrows with triangle) or fragmentation (arrows), were calculated. (D) Caspase-3/-7 activity of SACC-83 cells after the different treatments. All data are presented as the mean $\pm \mathrm{SD}$ of at least three independent experiments; ${ }^{*} \mathrm{P}<0.05$ vs. the control group; ${ }^{\sharp} \mathrm{P}<0.05$ vs. the celecoxib or tubastatin A groups $(n=3)$.

K163R mutant form of PTEN (CDI=1.0). Correspondingly, the synergistic upregulation of membrane-bound PTEN and downregulation of phospho-AKT was observed in the U-87
MG cells stably transfected with wild-type PTEN, but not the K163R mutant (Fig. 7B). Similar findings were observed with individual celecoxib or tubastatin A treatments, which slightly 
A

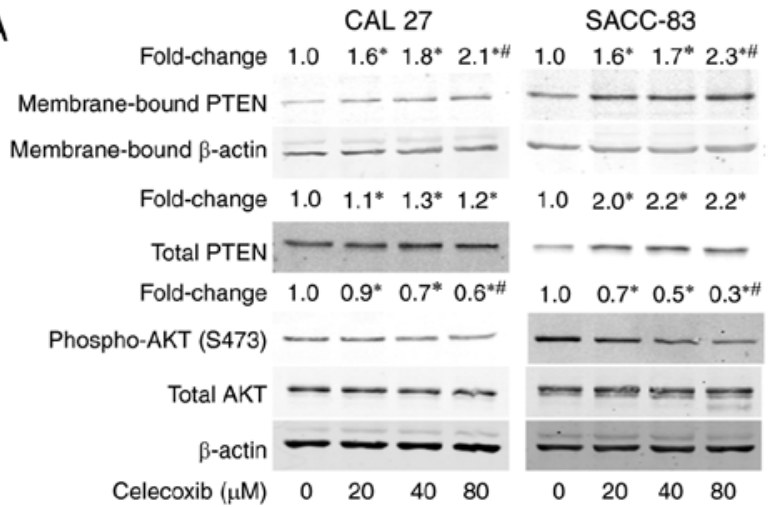

$\mathrm{B}$

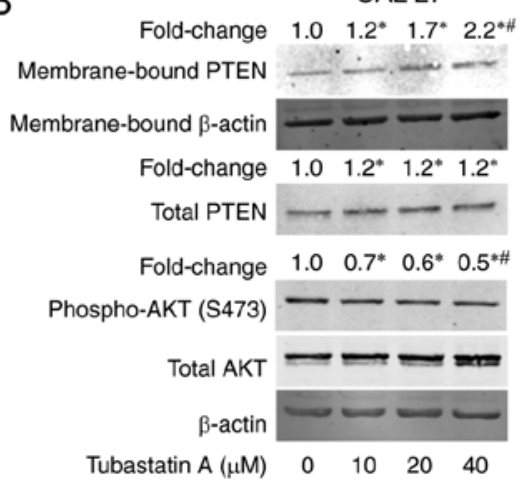

SACC-83

$1.01 .4^{*} 1.5^{*} 1.5^{*}$

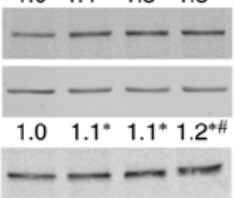

$\begin{array}{llll}1.0 & 0.9 * 0.8^{*} 0.7^{* *}\end{array}$

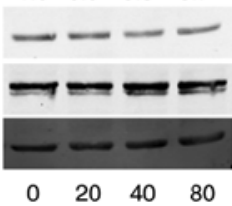

Figure 4. Treatment with the COX-2 inhibitor celecoxib or the HDAC6 inhibitor tubastatin A activates PTEN and inactivates AKT. (A) Western blot analysis of AKT and PTEN in the CAL 27 and SACC-83 cells following treatment with various doses of celecoxib for $24 \mathrm{~h}$. Cells were lysed and total or membrane proteins were extracted. (B) Western blot analysis of AKT and PTEN in the CAL 27 and SACC-83 cells after treatment with various doses of tubastatin A for $24 \mathrm{~h}$. Cells were lysed and total or membrane proteins were extracted. All data are presented as the mean $\pm \mathrm{SD}$ of at least three independent experiments; ${ }^{*} \mathrm{P}<0.05$ vs. the control group; ${ }^{"} \mathrm{P}<0.05$ vs. the 20 or $40 \mu \mathrm{M}$ groups $(\mathrm{n}=3)$.

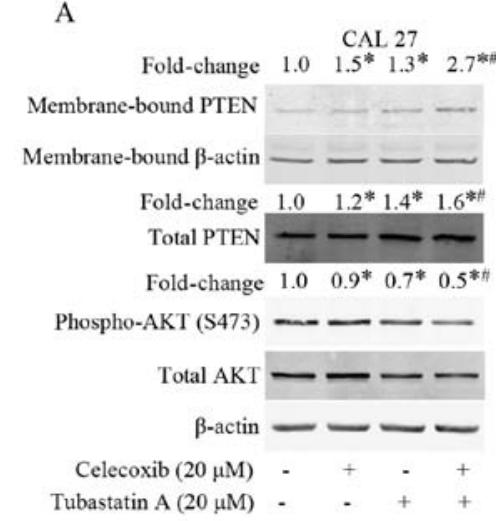

SACC-83 $1.0 \quad 1.7 * 1.2 * 2.4^{*}$
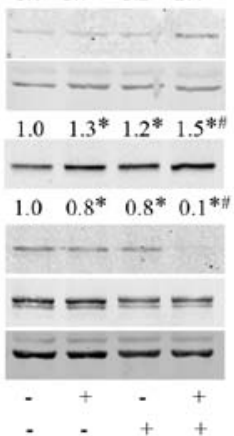

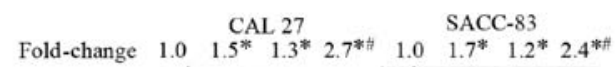
Membrane-bound PTEN _ _ _ _ _ _ _ _ _ Membrane-bound $\beta$-actin $\square-\square-\square-\square$ Fold-change $1.0 \quad 1.2^{*} \quad 1.2^{*} \quad 1.6^{* *} \quad 1.0 \quad 1.3^{*} \quad 1.2^{*} \quad 1.5^{* *}$ Total PTEN - - - - - - - -

Fold-change $1.0 \quad 0.9^{*} \quad 0.9^{*} 0.1^{* \#} \quad 1.0 \quad 0.9^{*} \quad 0.6^{*} \quad 0.3^{* *}$

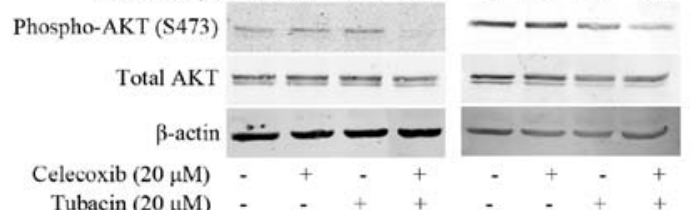

Figure 5. Effects of the combined treatment with an HDAC6 and a COX-2 inhibitors on PTEN and AKT. (A) Western blot analysis of AKT and PTEN after the different treatments. CAL 27 and SACC-83 cells were treated with celecoxib and/or tubastatin A. (B) CAL 27 and SACC-83 cells were treated with celecoxib and/or tubacin. Cells were lysed and total or membrane proteins were extracted. All data are presented as the mean \pm SD of at least three independent experiments; ${ }^{\mathrm{P}}<0.05$ vs. the control group; ${ }^{\prime} \mathrm{P}<0.05$ vs. the celecoxib or tubastatin A groups $(\mathrm{n}=3)$.

A

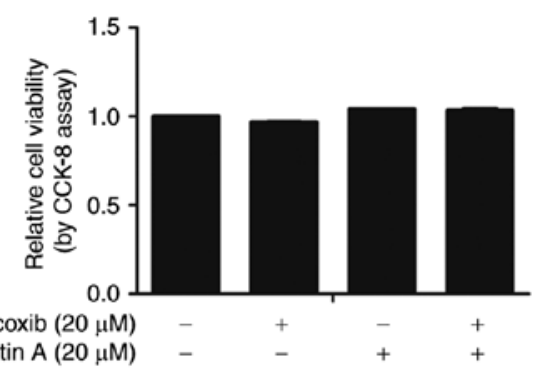

B

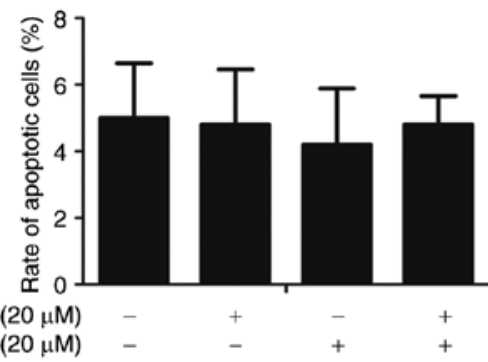

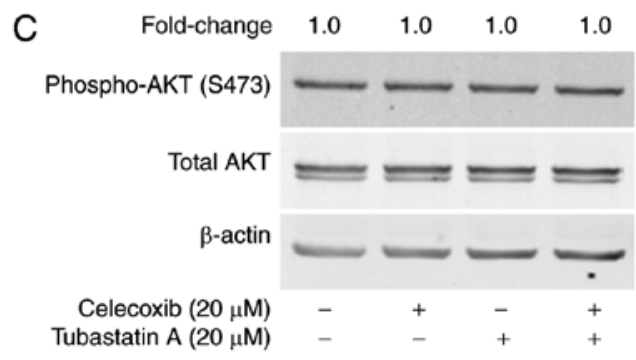

Figure 6. Combined treatment with the COX-2 inhibitor celecoxib and the HDAC6 inhibitor tubastatin A does not synergistically inhibit the proliferation of PTEN-deficient U-87 MG cells. (A) Cell proliferation was evaluated by CCK-8 assay after the different treatments. (B) Apoptosis was evaluated by DAPI staining after the different treatments. Data are presented as the mean \pm SD of at least three independent experiments. (C) Western blot analysis of AKT after the different treatments. 


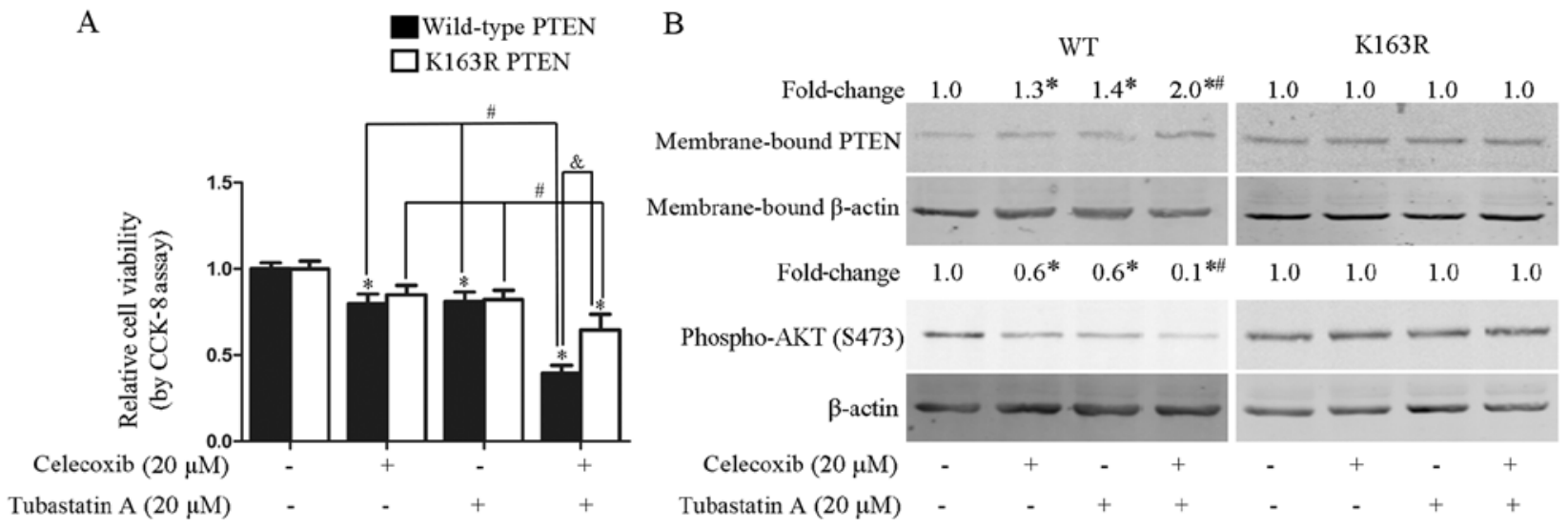

Figure 7. Combined treatment with the COX-2 inhibitor celecoxib and the HDAC6 inhibitor tubastatin A synergistically inhibits the proliferation of U-87 MG cells transfected with wild-type PTEN, but not those transfected with mutant-K163R. U-87 MG cells were stably transfected with wild-type PTEN or mutant PTEN-K163R. (A) Proliferation of stably transfected cells after the different treatments. The K163R mutation abolishes the HDAC6 inhibitor-induced acetylation and membrane translocation of PTEN. Cell proliferation was evaluated by CCK- 8 assay. All data are presented as the mean \pm SD of at least three independent experiments; ${ }^{\text {}} \mathrm{P}<0.05$ vs. the control group; ${ }^{~} \mathrm{P}<0.05$ vs. the control group; ${ }^{\prime} \mathrm{P}<0.05$ vs. the celecoxib or tubastatin $\mathrm{A}$ groups; ${ }^{\text {\& }} \mathrm{P}<0.05$ ( $\mathrm{n}=3$ ). (B) Western blot analysis of PTEN and AKT after the different treatments. U-87 MG cells stably transfected with wild-type PTEN or K163R mutant were treated with celecoxib and/or tubastatin A.
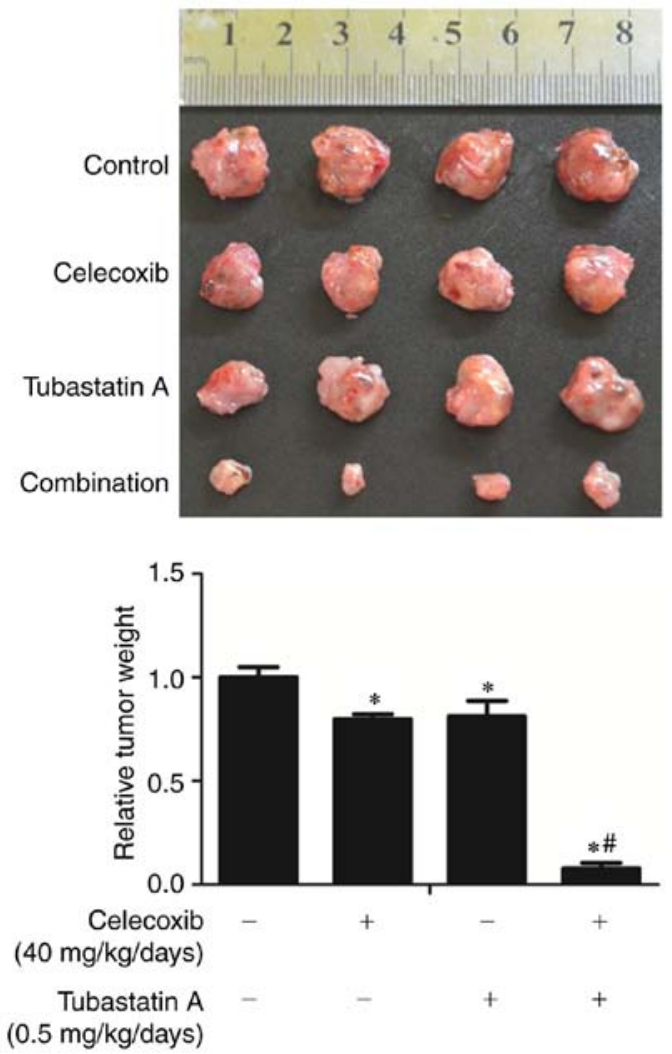

Figure 8. Combined treatment with the HDAC6 inhibitor tubastatin A and the COX-2 inhibitor celecoxib synergistically inhibits xenograft tumor growth. Images and weights of xenograft tumors derived from CAL 27 cells are presented. ${ }^{*} \mathrm{P}<0.05$ vs. the control group; ${ }^{\prime} \mathrm{P}<0.05$ vs. the celecoxib or tubastatin A groups $(n=4)$.

upregulated membrane-bound PTEN and downregulated phospho-AKT in the cells transfected with wild-type PTEN, but not in those transfected with mutant PTEN-K163R.

Combined treatment with inhibitor tubastatin $A$ and celecoxib synergistically inhibits xenograft tumor growth. To further confirm the synergistic antitumor effects of the HDAC6 inhibitor tubastatin A and the COX-2 inhibitor celecoxib in vivo, nude mice were inoculated with CAL 27 cells for 10 days, and the mice were then treated for three weeks with celecoxib and/or tubastatin A, or with the carriers as a control. As shown in Fig. 8, the weights of the xenograft tumors were significantly lower in the combined-treatment group than that noted in the other groups.

\section{Discussion}

In the present study, we demonstrated that combining an HDAC6 inhibitor with a COX-2 inhibitor produced synergistic antitumor effects. Our observations suggest a potential novel clinical strategy using HDAC6 inhibitors in combination with COX-2 inhibitors for antitumor therapy.

The combined treatment resulted in synergistic antitumor effects by activation of PTEN and inactivation of AKT. The combination of the HDAC6 inhibitor tubastatin A and the COX-2 inhibitor celecoxib induced apoptosis and inhibited proliferation, migration and invasion to a greater extent than either of the treatments alone in CAL 27 and SACC- 83 cells. The synergistic antitumor effects achieved by the combined treatment were further confirmed in nude mice with CAL 27 tumor xenografts. The combined-treatment group showed significantly decreased xenograft tumor weights than each of the single-treatment groups. The combination of tubastatin A and celecoxib achieved these affects by activating PTEN and inactivating AKT, which was demonstrated by the significant upregulation of membrane-bound PTEN (the activated form), and the corresponding significant downregulation of phosphoAKT, in the combined-treatment group compared with either of the single-treatment groups. Moreover, the combination of tubastatin A and celecoxib failed to produce synergistic inhibition of proliferation in PTEN-deficient U-87 MG cells, whereas the synergistic inhibition of proliferation was rescued in U-87 MG cells that had been stably transfected with wild-type PTEN, but not those transfected with mutant PTEN-K163R. In our previous study, it was demonstrated that wild-type PTEN could 
be acetylated and activated by a HDAC6 inhibitor, whereas the acetylation and activation of the mutant PTEN-K163R could not be induced by an HDAC6 inhibitor (17). Consistently, the levels of membrane-bound PTEN and phospho-AKT in the combined-treatment group were significantly upregulated and downregulated, respectively, in the U-87 MG cells stably transfected with wild-type PTEN, but not the mutant PTEN-K163R. Therefore, these results suggest that PTEN is responsible for the synergistic antitumor effects achieved by combination treatment with HDAC6 and COX-2 inhibitors.

To the best of our knowledge, these results demonstrated for the first time that COX-2 inhibitors are important agents for enhancing the antitumor effects of HDAC6 inhibitors. Therefore, these results provide the rationale for the use of combined HDAC6 inhibitors and COX-2 inhibitors in cancer chemotherapy. However, it should be noted that the combination of HDAC6 inhibitors and COX-2 inhibitors may be better applied in tumors that do not harbor aberrant or deleted PTEN. Otherwise, the use of this combination may fail to generate synergistic antitumor effects.

It was previously demonstrated that the upregulation of PTEN protein expression by COX-2 inhibitors was partially achieved through the downregulation of Sp1, whereas the upregulation of membrane-bound PTEN by COX-2 inhibitors is due to inhibition of phosphorylation (S380/T382/T383) in the PTEN C-terminal tail, as well as the upregulation of PTEN protein expression (22). Sp1 is an important negative regulator of PTEN that acts by recruiting HDAC1 to the core promoter of PTEN. Therefore, knockdown of Sp1 upregulates PTEN mRNA and protein expression (32). Moreover, COX-2 inhibitors downregulate $\mathrm{Spl}$ protein expression by promoting the degradation of Sp1 (33). These previous studies suggest that the underlying mechanism of COX-2 inhibitor-induced upregulation of membrane-bound PTEN relies partially on the downregulation of Sp1, and partially on reduced PTEN phosphorylation. Correspondingly, the present study found that celecoxib upregulated total PTEN and slightly upregulated the membrane-bound PTEN in CAL 27 and SACC-83 cells. The upregulation of membrane-bound PTEN in U-87 MG cells stably transfected with wild-type PTEN may be due to the downregulation of PTEN phosphorylation, as the total exogenous PTEN was not affected by the COX-2 inhibitor. However, surprisingly, celecoxib did not affect membranebound PTEN in U-87 MG cells stably transfected with mutant PTEN-K163R. Currently, we do not have an explanation for this phenomenon. We speculate that abolishing acetylation at K163 may somehow interfere with the regulation of PTEN membrane translocation by phosphorylation at the C-terminal tail. Further study is required to test this hypothesis.

In conclusion, the present study demonstrated that the combination of an HDAC6 inhibitor and a COX-2 inhibitor produced a synergistic antitumor effects through activation of PTEN and inactivation of AKT. Application of this combination may be suitable for the treatment of tumors without PTEN mutations or deletions.

\section{Acknowledgements}

The present study was supported by the National Natural Science Foundation of China (grant no. 81472764).

\section{References}

1. Liu T, Liu X and Li W: Tetrandrine, a Chinese plant-derived alkaloid, is a potential candidate for cancer chemotherapy. Oncotarget 7: 40800-40815, 2016.

2. Volm M and Efferth T: Prediction of Cancer Drug Resistance and Implications for Personalized Medicine. Front Oncol 5: 282, 2015.

3. Damaskos C, Karatzas T, Nikolidakis L, Kostakis ID, Karamaroudis S, Boutsikos G, Damaskou Z, Kostakis A and Kouraklis G: Histone deacetylase (HDAC) inhibitors: Current evidence for therapeutic activities in pancreatic cancer. Anticancer Res 35: 3129-3135, 2015.

4. Bukowska B, Gajek A and Marczak A: Two drugs are better than one. A short history of combined therapy of ovarian cancer. Contemp Oncol 19: 350-353, 2015.

5. Drazic A, Myklebust LM, Ree R and Arnesen T: The world of protein acetylation. Biochim Biophys Acta 1864: 1372-1401, 2016.

6. Zhang C, Zhong JF, Stucky A, Chen XL, Press MF and Zhang X: Histone acetylation: novel target for the treatment of acute lymphoblastic leukemia. Clin Epigenetics 7: 117, 2015.

7. Mathias RA, Guise AJ and Cristea IM: Post-translational modifications regulate class IIa histone deacetylase (HDAC) function in health and disease. Mol Cell Proteomics 14: 456-470, 2015.

8. Zhang H, Shang YP, Chen HY and Li J: Histone deacetylases function as novel potential therapeutic targets for cancer. Hepatol Res: Jul 26, 2016 (Epub ahead of print). doi: 10.1111/hepr.12757.

9. Gan YH and Zhang S: PTEN/AKT pathway involved in histone deacetylases inhibitor induced cell growth inhibition and apoptosis of oral squamous cell carcinoma cells. Oral Oncol 45: e150-e154, 2009.

10. Huang WJ, Liang YC, Chuang SE, Chi LL, Lee CY, Lin CW, Chen AL, Huang JS, Chiu CJ, Lee CF, et al: NBM-HD-1: A novel histone deacetylase inhibitor with anticancer activity. Evid Based Complement Alternat Med 2012: 781417, 2012.

11. Manal M, Chandrasekar MJ, Gomathi Priya J and Nanjan MJ: Inhibitors of histone deacetylase as antitumor agents: A critical review. Bioorg Chem 67: 18-42, 2016.

12. Ossenkoppele GJ, Lowenberg B, Zachee P, Vey N, Breems D, Van de Loosdrecht AA, Davidson AH, Wells G, Needham L, Bawden L, et al: A phase I first-in-human study with tefinostat - a monocyte/macrophage targeted histone deacetylase inhibitor - in patients with advanced haematological malignancies. Br J Haematol 162: 191-201, 2013.

13. Witt O, Deubzer HE, Milde T and Oehme I: HDAC family: What are the cancer relevant targets? Cancer Lett 277: 8-21, 2009.

14. Zhang Y, Kwon S, Yamaguchi T, Cubizolles F, Rousseaux S, Kneissel M, Cao C, Li N, Cheng HL, Chua K, et al: Mice lacking histone deacetylase 6 have hyperacetylated tubulin but are viable and develop normally. Mol Cell Biol 28: 1688-1701, 2008.

15. McConkey DJ, White M and Yan W: HDAC inhibitor modulation of proteotoxicity as a therapeutic approach in cancer. Adv Cancer Res 116: 131-163, 2012.

16. Saji S, Kawakami M, Hayashi S, Yoshida N, Hirose M, Horiguchi S, Itoh A, Funata N, Schreiber SL, Yoshida M, et al: Significance of HDAC6 regulation via estrogen signaling for cell motility and prognosis in estrogen receptor-positive breast cancer. Oncogene 24: 4531-4539, 2005.

17. Meng Z, Jia LF and Gan YH: PTEN activation through K163 acetylation by inhibiting HDAC6 contributes to tumour inhibition. Oncogene 35: 2333-2344, 2016.

18. Gu J, Wang D, Zhang J, Zhu Y, Li Y, Chen H, Shi M, Wang X, Shen B, Deng X, et al: GFR $\alpha 2$ prompts cell growth and chemoresistance through down-regulating tumor suppressor gene PTEN via Mir-17-5p in pancreatic cancer. Cancer Lett 380: 434-441, 2016.

19. Milella M,Falcone I, Conciatori F, Cesta Incani U,Del Curatolo A, Inzerilli N, Nuzzo CM, Vaccaro V, Vari S, Cognetti F, et al: PTEN: Multiple functions in human malignant tumors. Front Oncol 5: 24, 2015.

20. Tesio M, Trinquand A, Macintyre E and Asnafi V: Oncogenic PTEN functions and models in T-cell malignancies. Oncogene 35: 3887-3896, 2016.

21. Vazquez F, Matsuoka S, Sellers WR, Yanagida T, Ueda M and Devreotes PN: Tumor suppressor PTEN acts through dynamic interaction with the plasma membrane. Proc Natl Acad Sci USA 103: 3633-3638, 2006. 
22. Meng $\mathrm{Z}$ and Gan YH: Activating PTEN by COX-2 inhibitors antagonizes radiation-induced $\mathrm{AKT}$ activation contributing to radiosensitization. Biochem Biophys Res Commun 460: 198-204, 2015.

23. Ke J, Yang Y, Che Q, Jiang F, Wang H, Chen Z, Zhu M, Tong H, Zhang $\mathrm{H}$, Yan X, et al: Prostaglandin E2 (PGE2) promotes proliferation and invasion by enhancing SUMO-1 activity via EP4 receptor in endometrial cancer. Tumour Biol 37: 12203-12211, 2016.

24. Gan L, Qiu Z, Huang J, Li Y, Huang H, Xiang T, Wan J, Hui T, Lin $\mathrm{Y}, \mathrm{Li} \mathrm{H}$, et al: Cyclooxygenase-2 in tumor-associated macrophages promotes metastatic potential of breast cancer cells through Akt pathway. Int J Biol Sci 12: 1533-1543, 2016.

25. Salehifar E and Hosseinimehr SJ: The use of cyclooxygenase-2 inhibitors for improvement of efficacy of radiotherapy in cancers. Drug Discov Today 21: 654-662, 2016.

26. Vosooghi M and Amini M: The discovery and development of cyclooxygenase-2 inhibitors as potential anticancer therapies. Expert Opin Drug Discov 9: 255-267, 2014.

27. Liu R, Xu KP and Tan GS: Cyclooxygenase-2 inhibitors in lung cancer treatment: Bench to bed. Eur J Pharmacol 769: 127-133, 2015.

28. Wang X, Li G, Wang A, Zhang Z, Merchan JR and Halmos B: Combined histone deacetylase and cyclooxygenase inhibition achieves enhanced antiangiogenic effects in lung cancer cells. Mol Carcinog 52: 218-228, 2013
29. Gioanni J, Fischel JL, Lambert JC, Demard F, Mazeau C, Zanghellini E, Ettore F, Formento P, Chauvel P, Lalanne CM, et al: Two new human tumor cell lines derived from squamous cell carcinomas of the tongue: Establishment, characterization and response to cytotoxic treatment. Eur J Cancer Clin Oncol 24: $1445-1455,1988$.

30. Li SL: Establishment of a human cancer cell line from adenoid cystic carcinoma of the minor salivary gland. Zhonghua Kou Qiang Yi Xue Za Zhi 25: 29-31, 62, 1990 (In Chinese).

31. Wu Y, Zhu Y, Li S, Zeng M, Chu J, Hu P, Li J, Guo Q, Lv XB and Huang G: Terrein performs antitumor functions on esophageal cancer cells by inhibiting cell proliferation and synergistic interaction with cisplatin. Oncol Lett 13: 2805-2810, 2017.

32. Kou XX, Hao T, Meng Z, Zhou YH and Gan YH: Acetylated Sp1 inhibits PTEN expression through binding to PTEN core promoter and recruitment of HDAC1 and promotes cancer cell migration and invasion. Carcinogenesis 34: 58-67, 2013.

33. Abdelrahim M and Safe S: Cyclooxygenase-2 inhibitors decrease vascular endothelial growth factor expression in colon cancer cells by enhanced degradation of Spl and Sp4 proteins. Mol Pharmacol 68: 317-329, 2005. 